\title{
Erratum to: Myocardial Infarction Detection from Left Ventricular Shapes Using a Random Forest
}

\author{
Jack Allen ${ }^{1(凶)}$, Ernesto Zacur ${ }^{2}$, Erica Dall'Armellina ${ }^{1}$, Pablo Lamata ${ }^{2}$, \\ and Vicente Grau ${ }^{1}$ \\ ${ }^{1}$ University of Oxford, Oxford, UK \\ jack.allen@jesus.ox.ac.uk \\ 2 King's College London, London, UK
}

Erratum to:

Chapter 20: O. Camara et al. (Eds.)

Statistical Atlases and Computational Models of the Heart, DOI: 10.1007/978-3-319-28712-6_20

The following acknowledgement was omitted from the paper entitled "Myocardial Infarction Detection from Left Ventricular Shapes Using a Random Forest"

Acknowledgements. This work was supported by funding from the Medical Research Council (MRC) and Engineering and Physical Sciences Research Council (EPSRC) [grant number EP/L016052/1].

The updated original online version for this chapter can be found at 10.1007/978-3-319-28712-6_20 